treatment, and rapid disappearance in others. Antibodies were discovered in some people who had no history or evidence of anthrax. This pattern has been reported previously: 11 out of 72 otherwise healthy workers in a goat hair mill were found to have antibodies. ${ }^{\circ}$ Recovery from anthrax is said to be followed by prolonged immunity and recurrences are said to be extremely rare, ${ }^{8}$ but we have not confirmed this in the Gambia. Serological examination showed that though antibodies may persist for 12 months (possibly as a result of repeated subinfective doses or subclinical attacks) there was at least one authenticated recurrence within 12 months of primary infection, which corresponded with the laboratory findings that this strain contains a weak antigen.

Unlike the mortality in Iran, ${ }^{1}$ in the Gambia mortality was not related to any particular site, to the multiplicity of lesions, or to the extent of local inflammatory response. We did not confirm either that mortality and morbidity were related to the nutritional state of the people; on the contrary, their nutritional state was at its best at the time of maximum attack.

How can this disease be eradicated ? It is not limited to the Gambia, being present in the adjoining area of Senegal. It is firmly established in animals, particularly sheep and goats, and it would be impossible to immunize these effectively. Humans could be immunized, ${ }^{10}$ but three or four injections are needed to give even temporary immunity. An international organization would have to carry out an immunization programme and organize the necessary annual booster doses. Nevertheless, even temporary immunity may break the cycle by allowing a natural reduction of spore counts in the soil. Public health measures to ensure the correct disposal of animal carcases and the control of vultures may also help. An attempt could be made to change the habit of using the palm-fibre loofah, to limit its use to one per person, and to advise disposal of the loofah before it became contaminated with anthrax spores.

There is no evidence to suggest that the disease is declining in severity or prevalence, and from what is known of the antigenicity of this organism it is most unlikely that natural or "herd immunity" will protect the population even when they are repeatedly exposed to the disease. The converse may be true unless active steps are taken to check or eradicate the disease before it becomes endemic and spreads to other villages and areas of the country.

We thank Dr. I. A. McGregor and Dr. W. Bray, directors of the M.R.C. Laboratories in the Gambia; Professor R. J. Harris, director of the M.R.E., Porton Down; Dr. P. N'Dow, director of medical services in the Gambia, Dr. W. N'Dow, director of veterinary services in the Gambia; Dr. F. Oldfield, pathologist, Royal Victoria Hospital, the Gambia; Professor Collard and his staff at the microbiology department, University of Manchester; and Dr. N. Dodd, department of tropical community medicine, Liverpool, for help with the analyses of cases.

\section{References}

1 Amidi, S., et al., Zeitschrift für Tropenmedizin und Parasitologie, 1974, 25, 96.

2 Wilson, G. S., and Miles, A. A., Topley qnd Wilson's Principles of Bacteriology and Immunity, 6th edn., p. 2208. London, Arnold, 1975.

${ }^{3}$ McGregor, I. A., et al., Transactions of the Royal Society of Tropical Medicine and Hygiene, 1970, 64, 48.

4 Davies, D. G., Fournal of Hygiene, 1960, 58, 177

5 Fendall, N. R. E., and Grounds, J. G., fournal of Tropical Medicine and Hygiene, 1965, 68, 77.

- Kohout, E., Sehat, A., and Ashraf, M., American fournal of the Medical Sciences, 1964, 3, 565.

7 Sen, S. K., and Minett, F. C., Indian fournal of Veterinary Science and Animal Husbandry, 1944, 14, 149.

${ }^{8}$ Elkin, I. I., A Course in Epidemiology, p. 491. Oxford, Pergamon Press, 1961.

- Norman, P. S., et al., American fournal of Hygiene, 1960, 72, 32.

10 Darlow, H. M., Belton, F. C. and Henderson, D. W., Lancet, 1956, 2, 476.

\title{
Effect of breast-feeding on pituitary-ovarian function after childbirth
}

\author{
J BONNAR, M FRANKLIN, P N NOTT, A S MCNEILLY
}

British Medical fournal, 1975, 4, 82-84

\section{Summary}

Pituitary and ovarian function at the end of pregnancy and during the first six weeks after delivery was investigated serially in women who fully breast-fed their infants and in women who did not. In the women who did not breast-feed the plasma prolactin level decreased rapidly and from the third day after delivery was significantly lower than in the breast-feeding mothers, reaching

Nuffield Department of Obstetrics, University of Oxford, John Radcliffe Hospital, Oxford

J BONNAR, MD, FRCOG, clinical reader now professor of obstetrics and gynaecology, Trinity College Medical School, Dublin.

University Department of Psychiatry, Oxford

M FRANKLIN, LRIC, senior technician

$P$ N NOTT, MRCP, lecturer

Department of Chemical Pathology, St. Bartholomew's Hospital, London

A S MCNEILLY, PH D, research lecturer the normal range of the menstrual cycle by the third week of the puerperium. In the breast-feeding mothers the plasma prolactin was still raised six weeks after delivery. The levels of FSH in both groups were identical and increased over the third week of the puerperium. Plasma oestrogen fell steeply in both groups during the first two weeks after delivery. In the breast-feeding mothers plasma oestrogen remained depressed but increased in the non-lactating women, reflecting follicular development in the ovary in response to FSH; the plasma oestrogen levels were significantly higher in the nonlactating women from the 17th day of the puerperium onwards. These findings support the concept that in breast-feeding women prolactin delays the return of ovulation by inhibiting the ovarian response to FSH stimulation.

\section{Introduction}

Breast-feeding postpones the return of ovulation and menstruation after childbirth, and postpartum amenorrhoea is related to the duration of breast-feeding. ${ }^{1-4}$ Studies on the influence of lactation on ovulation have shown that breast-feeding women 
have little chance of becoming pregnant until the infant is over 10 weeks old, but in women who do not breast-feed ovulation can occur as early as the fourth week post partum. ${ }^{5}$ To provide more information on the endocrine effects of breast-feeding we undertook a serial study of pituitary and ovarian hormones in women who fully breast-fed their infants and compared these results with the values in mothers who did not breast-feed their infants. Plasma follicle-stimulating hormone (FSH), luteinising hormone (LH), oestrogen, progesterone, prolactin, and human chorionic gonadotrophin (HCG) were studied in late pregnancy and for the first six weeks after delivery.

\section{Patients and methods}

The study was explained to healthy women in late pregnancy and 60 women volunteered to take part. Serial studies proved feasible in 15 women who did not breast-feed, nine women who fully breast-fed up to or beyond the sixth-week after delivery, and three women who breast-fed for only three weeks. All had normal pregnancies and vaginal delivery of term infants and had no hormone or other drug treatment apart from analgesics in labour and in the first week after delivery. Three blood samples were taken between 36 and 39 weeks' gestation, and after delivery blood samples were taken on the first day, every second day for the first week, and thereafter every third day until the end of the sixth week. In the first week of the puerperium blood samples were taken between 9 and $11 \mathrm{am}$. After the patients left hospital blood samples were taken in the patient's home between 9 am and 12 noon. The blood was collected into heparinised tubes, and after separation the plasma was stored at $-20^{\circ} \mathrm{C}$ until assayed. All the samples from each patient were assayed at the same time.

\section{HORMONE ASSAYS}

All assays were performed in duplicate.

Plasma LH and FSH were measured by HCG and FSH double antibody radioimmunoassay. ${ }^{6}$ The second international reference preparation of human menopausal gonadotrophin (2nd IRP-HMG) with a potency of $40 \mathrm{IU} /$ ampoule was used as a standard. Dilutions of this standard made up in either phosphate buffer or plasma obtained from hypophysectomised subjects showed satisfactory parallelism when compared with dilutions of MRC LH 68/40 pituitary standard (potency, $40 \mathrm{U} /$ ampoule $\mathrm{LH}$ ) and postmenopausal plasma: $1 \mathrm{mU}$ MRC LH 68/40 was equivalent to $5 \mathrm{mIU}$ 2nd IRP-HMG. The antibody to FSH showed less than $1 \%$ cross-reaction with purified LH. The sensitivity for the LH assay was $1 \mathrm{mIU}$ 2nd IRP-HMG/ml and 2 mIU 2nd IRP-HMG for the FSH assay.

Plasma total oestrogens and progesterone were estimated by a modification of the radioimmunoassay method of Hotchkiss. ${ }^{7}$ The antibody used in the total oestrogen assay was raised against oestradiol-17 $\beta$ and had the following cross-reactions: $100 \%$ oestradiol- $17 \beta, 40 \%$ oestradiol- $17 \alpha, 35 \%$ oestrone, and $8 \%$ oestriol. The only substance found to cross-react with the progesterone antibody was deoxycorticosterone $(25 \%)$; all others tested were $<0.5 \%$.

Plasma prolactin was measured by a homologous double antibody radioimmunoassay using highly purified human prolactin for standards and iodination, as described elsewhere. ${ }^{8}$ The sensitivity for the prolactin assay was $1.6 \mathrm{ng}$.

Plasma HCG $\beta$-subunit was estimated by a double antibody radioimmunoassay similar to that used for plasma prolactin. ${ }^{\circ}$ The antibody used (National Pituitary Agency, NPA-SB6) was specific for HCG- $\beta$ $(100 \%)$ and cross-reacted with HCG- $\alpha(3 \%)$ only. All other hormones and subunits showed $<0 \cdot 1 \%$ cross-reaction.

Analysis of results-Logarithmic transformation of the plasma FSH, LH, HCG, and oestrogen results was used for statistical analysis with Student's $t$ test

\section{Results}

The mean plasma levels of prolactin, $\mathrm{FSH}$, and oestrogen in the mothers who breast-fed for at least six weeks and the non-lactating mothers are shown in fig 1 . At the 37th-38th week of pregnancy plasma prolactin, FSH, and oestrogen were similar in both groups. After delivery the plasma prolactin level in the non-lactating mothers decreased ranidly and was significantly lower than in the breast- feeding women from the third day of the puerperium. The plasma prolactin in the non-lactating women fell to the range of the normal menstrual cycle by the end of the third week of the puerperium; eight of the 15 women had prolactin levels below $25 \mu \mathrm{g} / \mathrm{l}$ by the end of the second week after delivery. In the lactating women the prolactin levels were still raised at the sixth week.

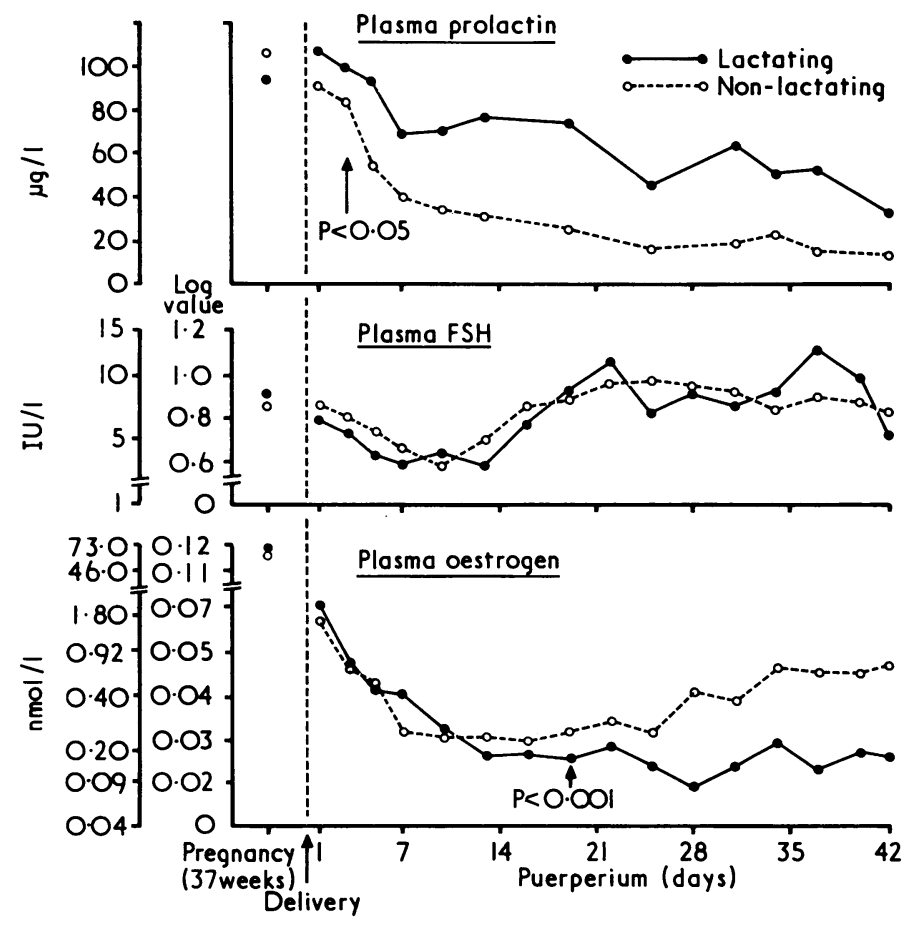

FIG 1-Comparison of serial levels of plasma prolactin, FSH, and osetrogen in last three weeks of pregnancy and first six weeks after delivery in nine mothers who fully breast-fed and 15 non-lactating mothers. Values for plasma FSH and plasma oestrogen were not a normal distribution and logarithmic transformation of data was used for statistical analysis.

Conversion: SI to Traditional Units-Oestrogen (oestradiol): $1 \mathrm{nmol} / 1 \approx$ $27 \cdot 2 \mathrm{ng} / 100 \mathrm{ml}$.

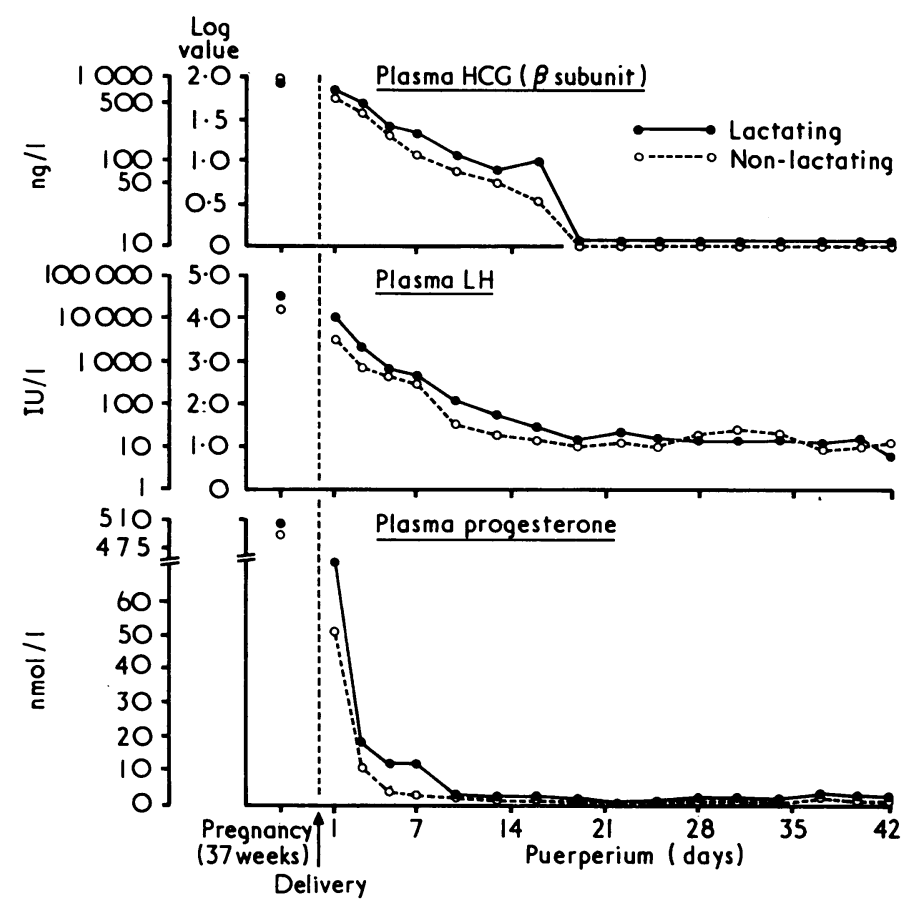

FIG 2-Comparison of plasma HCG ( $\beta$-subunit), LH, and progesterone in nine lactating and 15 non-lactating mothers.

Conversion: SI to Traditional Units-Progesterone: $1 \mathrm{nmol} / 1 \approx 0.32 \mathrm{ng} / \mathrm{ml}$. 
The levels of plasma FSH in both groups were almost identical and fell during the first two weeks of the peurperium and increased from the 14th to the 21st day after delivery to levels in the upper limit of the range for the follicular phase of the menstrual cycle. Plasma oestrogen in both groups fell steeply during the first two weeks after delivery and remained depressed in the patients who were breast-feeding. In the non-lactating group oestrogen levels increased from the third week onwards and were significantly higher that in breast-feeding women from the 17 th day of the puerperium.

The mean plasma levels of HCG ( $\beta$-subunit), $\mathrm{LH}$, and progesterone are shown in fig 2 . The levels of HCG- $\beta$ were virtually undetectable by the end of the third week after delivery. The plasma LH activity followed the same pattern as HCG during the first three weeks after delivery but during the fifth week an increase of $\mathrm{LH}$ was found in the non-lactating group, with a rise occurring in 10 out of the 15 women; the distribution of the $\mathrm{LH}$ results was skewed and no significant difference from the LH levels in the breast-feeding women was found using logarithmic values. The plasma progesterone levels were similar in both groups except during the fifth week after devliery, when a slight increase of progesterone was found in three out of 12 nonlactating women and one woman who was breast-feeding.

In the three women who breast-fed for three weeks the prolactin levels were below $25 \mu \mathrm{g} / \mathrm{l}$ before the end of the fourth week after delivery and the oestrogen levels increased between the fifth and sixth week.

\section{Discussion}

During late pregnancy and the first two days of the puerperium levels of prolactin around $100 \mu \mathrm{g} / \mathrm{l}$ were found, which accords with other reports. ${ }^{1011}$ Thereafter a precipitate fall occurred in the non-lactating women, the prolactin falling to the normal non-pregnant range $(12-25 \mu \mathrm{g} / \mathrm{l})$ by the second or third week after delivery. In the women breast-feeding for six weeks or more plasma prolactin levels slowly decreased and at the sixth week the mean level was still above normal at $34.2 \mu \mathrm{g} / 1$ with a wide range (15-74 $\mu \mathrm{g} / \mathrm{l})$. The range of values reflects fluctuating levels in the mother as a result of the bursts of prolactin release that accompany breast-feeding. In the non-lactating women all the prolactin levels were within the normal non-pregnant range by the end of the third week of the puerperium. During the second and third weeks after delivery plasma FSH gradually increased to follicular phase levels, which accords with the findings of others. ${ }^{212} \mathrm{By}$ the third week after delivery the levels of plasma oestrogen in the non-lactating women began to increase, while those in the women who were breast-feeding remained low.

Our findings therefore indicate that plasma oestrogens do not increase in women who are fully breast-feeding despite the rise of FSH. This supports the view that the ovaries do not respond to FSH stimulation in the presence of increased prolactin levels. ${ }^{6} 7$ Prolactin has an affinity for ovarian tissue, ${ }^{13}$ and thus a local action may prevent follicular development in response to FSH stimulation. In concentrations above $30 \mu \mathrm{g} / \mathrm{l}$, a level below that seen in the breast-feeding women, prolactin reduces steroid hormone production from human granulosa cells in vitro. ${ }^{14}$ When exogenous gonadotrophins are given to women who are breast-feeding no change in ovarian steroid output can be detected. ${ }^{15}$ In the early post-partum period the LH and FSH response to gonadotrophin-releasing hormone is depressed and this lack of response is more prolonged in women who lactate. ${ }^{16}$ The periodic bursts of prolactin release that follow suckling may also therefore have an inhibitory effect on hypothalopituitary function. This, together with the inhibiting effect of prolactin on the ovary, would account for the suppression of ovulation and amenorrhoea in women who breast-feed. The return of ovarian activity in the non-lactating women was delayed for one to two weeks after the prolactin levels had declined. This was suggested by the absence of a rise in the plasma oestrogens until the end of the fourth week after delivery in the non-lactating women, although plasma FSH rose between the seventh and the 14th day.

The rise of plasma oestrogen in the non-lactating women indicates ovarian follicular response, and in two of the 15 women the level increased to that of the late follicular phase. In a significant proportion of women who do not breast-feed follicular development may therefore progress to a mature Graffian follicle and ovulation occur before the sixth week after delivery. Clinical studies on the effect of lactation without supplemental feeding have indicated that lactation provides effective birth control up to the 10 th week after delivery. ${ }^{35}$

Although full breast-feeding will postpone the return of menstruation and ovulation after delivery, suppression of ovulation is limited in time as the plasma prolactin decreases even with continuing lactation. Possibly pharmacologically induced increases in plasma prolactin might be valuable as a method of prolonging the suppression of ovulation in women who are breast-feeding. Increasing the prolactin level might also be of value in establishing successful lactation in women who encounter problems with breast-feeding. Despite the fact that prolactin serves many diverse functions in animals, its only confirmed action in humans is the initiation and continuance of lactation. ${ }^{16}$ Further research may show interesting possibilities for the use of prolactin, using its action on the ovary for the spacing of pregnancies.

We thank Professor Michael Gelder and Dr Pamela Mackinnon for their help with this study. The work was supported by a research grant from the Medical Research Council (G970/227C) to the University Department of Psychiatry and Nuffield Department of Obstetrics and Gynaecology. Expert clinical help was given by Sisters Turner and Armytage. Standard human prolactin was kindly provided by Dr H Friesen, and antisera as follows: progesterone by Dr B Burr, oestrogen by Dr G A Abraham, FSH by Professor W R Butt, and LH by Dr C A Paulsen. Full details of the hormone data will be provided on request. Reprints can be obtained from Professor J Bonnar, Rotunda Hospital, Dublin. We thank the women who took part in this investigation and willingly accepted the discomfort of multiple venepunctures in the interest of medical research.

\section{References}

${ }^{1}$ Berman, M L, Hanson, K, and Hellman, I L, American fournal of Obstetrics and Gynecology, 1972, 114, 524.

2 Reyes, F I, Winter, J S D, and Faiman, C, American fournal of Obstetrics and Gynecology, 1972, 114, 589.

${ }^{3}$ Perez, A, et al, American fournal of Obstetrics and Gynecology, 1972, 114, 1041.

4 Tietze, C, in Advances in Planned Parenthood, ed A J Sobrero and R M Harvey, p 122. Excerpta Medica, 1971.

5 Cronin, T J, Lancet, 1968, 2, 422.

${ }^{6}$ Midgley, A R, Fournal of Endocrinology, 1966, 79, 10.

${ }^{7}$ Hotchkiss, J, Atkinson, L E, and Knobil, E, Endocrinology, 1971, 89, 177.

${ }^{8}$ McNeilly, A S, and Chard, T, Clinical Endocrinology, 1974, 3, 105.

${ }^{9} \mathrm{McNeilly,} \mathrm{A} \mathrm{S,} \mathrm{and} \mathrm{Hagen,} \mathrm{C,} \mathrm{American} \mathrm{fournal} \mathrm{of} \mathrm{Obstetrics} \mathrm{and} \mathrm{Gyne-}$ cology, in press.

${ }^{10}$ Rolland, R, et al, Clinical Endocrinology, 1975, 4, 15.

11 Tyson, J E, and Friesen, H G, American fournal of Obstetrics and Gynecology, 1973, 116, 377.

12 Crystle, C D, Powell, J, and Vernon, V C, Obstetrics and Gynecology, 1970, $36,887$.

13 Turkington, R W, Frantz, W L, and Majumder, G C, International Symposium on Human Prolactin, p 41. Excerpta Medica, Amsterdam, 1973.

14 McNatty, K P, Sawyers, R S, and McNeilly, A S, Nature, 1974, 250, 653.

15 Zarate, A, et al, American fournal of Obstetrics and Gynecology, 1974, 112, 1130.

${ }^{16}$ Friesen, H G, Fournier, P, and Desjardin, P, Clinical Obstetrics and Gynecology, 1973, 116, 377. 